FACTA UNIVERSITATIS

Series: Mechanical Engineering Vol. 14, N 2, 2016, pp. 159 - 168

Original scientific paper

\title{
REDUCTION OF RESIDUAL SHEAR STRESS IN THE LOADED CONTACT USING FRICTION HYSTERESIS
}

\author{
UDC 531.4
}

\section{Adrian Kraft, Roman Pohrt}

Berlin Institute of Technology, Germany

\begin{abstract}
We investigate the tangential contact problem of a spherical indenter at constant normal force. When the indenter is subjected to tangential movement, frictional shear stresses arise at the interface and do not vanish when it is moved backwards. We study the evolution of shear stress when the indenter is moved back and forth at falling amplitude. The method of dimensionality reduction (MDR) is employed for obtaining the distribution of stick and slip zones as well as external forces and the final stress distribution. We find that the shear stress decreases. For the special case of linearly falling amplitude of the movement, we observe uniform peaks in the shear stress. The absolute value of the shear stress peaks is reduced best for a high number of back-andforth-movements with slowly decreasing amplitude.
\end{abstract}

Key Words: Coulomb Friction, Residual Stress, Contact Mechanics, Stick, Slip

\section{INTRODUCTION}

Loaded contacts exist in a broad variety of technical and natural situations. A basic frictional couple consists of two deformable bodies, which are pressed together and subjected to an additional tangential loading.

When the tangential load is below the threshold of gross sliding, some micro-slip occurs nevertheless. In technical contact that experience cyclic loading, the relative movement of the bodies is often invisible to the naked eye. However, the friction involved can cause effects such as damping [3,4] and fretting wear [8]. The same applies in a slightly more complicated fashion for biaxial loading [5].

Consider a contact where the normal load is held constant, so that the bodies cannot separate. Initially, the pure normal loading can take place without inducing shear stress in

Received February 24, 2016 / Accepted July 10, 2016

Corresponding author: Roman Pohrt

Technische Universität Berlin, Straße des 17. Juni 135, 10623 Berlin, Germany

E-mail: roman.pohrt@tu-berlin.de 
the contact interface. However, when some tangential load is applied, shear stress arises. Upon unloading, the stress does not simply vanish due to the dual nature of the contact (stick zones vs. slip zones). Tangential force and resulting tangential displacement together form a system with hysteresis, which can be described with a Preisach formalism [10]. In 1928, Prandtl developed a model for microtribology out of many micro-sliders, which also included hysteresis [12] (see [11] for English translation of the original paper). In his model, the exact state of the system at any moment of time depends on its prehistory and can be very complicated. Prandtl poses the question of whether it is possible to restore the virgin state. With very simple arguments, he shows that if the contact partners start to oscillate with large amplitude and the amplitude then decreases slowly, then each slider finally comes to a neutral, non-stressed position and the system returns to the virgin state. He compares this result with demagnetization through slowly decreasing oscillating magnetic fields, first studied by E. Madelung [6]. In this paper, we will apply this idea to the loaded contact with partial sliding.

Assume a typical Hertzian contact. A spherical indenter with radius $\mathrm{R}$ is pressed into a plane. The sphere is approximated by a parabola and we assume that the plane is rigid. We keep normal force $F_{N}$ that we first applied constant throughout the following. This configuration is known as the Hertzian contact with contact radius a and has been studied in great detail. Normal stress distribution $\mathrm{p}$ as a function of radial coordinate $\mathrm{r}$ reads

$$
\begin{aligned}
& \mathrm{p}(\mathrm{r})=\mathrm{p}_{\max }\left(1-\frac{\mathrm{r}^{2}}{\mathrm{a}^{2}}\right)^{1 / 2} \\
& \mathrm{p}_{\max }=\frac{\left(6 \mathrm{~F}_{\mathrm{N}}\right)^{1 / 3}}{\pi}\left(\frac{\mathrm{E}^{*}}{\mathrm{R}}\right)^{2 / 3}
\end{aligned}
$$

Here we used the reduced modulus of elasticity:

$$
\frac{1}{\mathrm{E}^{*}}=\frac{1-v_{1}^{2}}{\mathrm{E}_{1}}+\frac{1-v_{2}^{2}}{\mathrm{E}_{2}},
$$

for two elastic bodies with moduli of elasticity $E_{1}$ and $E_{2}$, shear moduli $G_{1}$ and $G_{2}$ and Poisson's ratios $v_{1}$ and $v_{2}$, respectively. The equivalent reduced shear modulus is:

$$
\frac{1}{\mathrm{G}^{*}}=\frac{1-v_{1}^{2}}{\mathrm{G}_{1}}+\frac{1-v_{2}^{2}}{\mathrm{G}_{2}} .
$$

We now move the indenter horizontally along one spatial direction and assume that Coulombs law of friction is valid in its simplest form $\tau(\mathrm{r}) \leq \mu \mathrm{p}(\mathrm{r})$. Here $\tau$ is the shear stress and $\mu$ is the coefficient of friction. This condition leads to the partitioning of the contact area into a stick area in the center and a slip area at the edges of it. For example, at the very edge of the contact surface, normal stress disappears and the slightest displacement of the indenter would lead to shear stress violating our friction condition if we assumed it would stick to the plane. The solution of this is that some parts of the contact area are being slipped over the plane while the others stick to it. At a certain point, the stick area completely disappears and the whole contact is slipping. Tangential displacement $\mathrm{u}_{\mathrm{x}}{ }^{(0)}$ at this last point reads [2]: 


$$
\mathrm{u}_{\mathrm{x} \max }^{(0)}=\mu \frac{\mathrm{E}^{*}}{\mathrm{G}^{*}} .
$$

Let us now drag the indenter back and forth along the same spatial direction on the rigid plane. We observe different shear stress distributions depending on the motion history. The shear stress created from the first movement will not disappear if we simply return to our starting point. In the following, we will analyze the shear stress under a back and forth movement with falling amplitude with the aim of reducing this stress. For the analysis of the system, the Aleshins method of memory diagrams is well suited. Because the contact stress distribution accumulates more and information of past reversal points, the HH-mode described in sec. 31 of [9] should be applied iteratively. Using careful algebra, an explicit analytical solution might be achievable. However, we decided to use the method of dimensionality reduction for our analysis, which we describe in the next section. Alternatively, one could employ a full Boundary Elements solution [7].

\section{MDR AND DISCRETIZED 1D-MODEL}

We consider the three-dimensional contact of two elastic bodies. In the following, we presuppose axially symmetric profiles. Let $\mathrm{z}=\mathrm{f}(\mathrm{r})$ be the difference between the profiles of the bodies.

According to the theorems of the method of dimensionality reduction (MDR), this contact can be exactly replaced by a contact with a one-dimensional linearly elastic foundation with independent springs.

To reduce the initial three-dimensional contact to a one-dimensional one, two steps are required. First, we replace the elastic bodies by the one-dimensional linearly elastic foundation. Normal stiffness $\Delta \mathrm{k}_{\mathrm{z}}$ and tangential stiffness $\Delta \mathrm{k}_{\mathrm{x}}$ of the springs are chosen according to:

$$
\begin{aligned}
& \Delta \mathrm{k}_{\mathrm{z}}=\mathrm{E}^{*} \Delta \mathrm{x} \\
& \Delta \mathrm{k}_{\mathrm{x}}=\mathrm{G}^{*} \Delta \mathrm{x},
\end{aligned}
$$

where $\Delta \mathrm{x}$ denotes the distance between two springs.

Second, we replace the three-dimensional profile $\mathrm{z}=\mathrm{f}(\mathrm{r})$ with a one-dimensional profile in accordance to [2]:

$$
g(x)=|x| \int_{0}^{|x|} \frac{f^{\prime}(r)}{\sqrt{x^{2}-r^{2}}} d r .
$$

The reverse transformation is:

$$
f(r)=\frac{2}{\pi} \int_{0}^{r} \frac{g(x)}{\sqrt{r^{2}-x^{2}}} d x .
$$

If now transformed profile $\mathrm{g}(\mathrm{x})$ is pressed with normal force $\mathrm{F}_{\mathrm{N}}$ and resulting indentation depth $\mathrm{d}$ into the elastic foundation, we obtain the displacement in the contact area:

$$
u_{\mathrm{z}}(\mathrm{x})=\mathrm{d}-\mathrm{g}(\mathrm{x})
$$


For non-adhesive contacts we can set:

$$
\mathrm{u}_{\mathrm{z}}( \pm \mathrm{a})=0 \Rightarrow \mathrm{d}:=\mathrm{g}(\mathrm{a}) \text {, }
$$

where a is the contact radius. We again denote the length a the 'contact radius' because following the theorems of MDR, all the lengths in the model are equal to the respective ones in a three-dimensional problem. The same applies to the contact area. The force of a spring at point $\mathrm{x}$ inside the contact area equals:

$$
\Delta \mathrm{F}_{\mathrm{z}}(\mathrm{x})=\Delta \mathrm{k}_{\mathrm{z}} \mathrm{u}_{\mathrm{z}}(\mathrm{x})=\mathrm{E}^{*} \mathrm{u}_{\mathrm{z}}(\mathrm{x}) \Delta \mathrm{x} .
$$

If we choose the spring separation distance to be infinitesimal, we get for the normal force:

$$
F_{N}:=E^{*} \int_{-a}^{a} u_{z}(x) d x=2 E^{*} \int_{0}^{a}(d-g(x)) d x .
$$

After bringing the elastic bodies in normal contact with force $\mathrm{F}_{\mathrm{N}}$, we apply tangential movement $\mathrm{u}_{\mathrm{x}}^{(0)}$ to the indenter and assume that Coulomb's law of friction is valid following:

$$
\begin{gathered}
\tau(\mathrm{r}) \leq \mu \mathrm{p}(\mathrm{r}) \text { for stick, } \\
\tau(\mathrm{r})=\mu \mathrm{p}(\mathrm{r}) \text { for slip. }
\end{gathered}
$$

Now every spring sticks to the indenter as long as $\Delta \mathrm{F}_{\mathrm{x}}=\Delta \mathrm{k}_{\mathrm{x}} \mathrm{u}_{\mathrm{x}}{ }^{(0)}<\mu \Delta \mathrm{F}_{\mathrm{z}}$. This results in the following conditions:

$$
\begin{aligned}
& \mathrm{u}_{\mathrm{x}}(\mathrm{x})=\mathrm{u}_{\mathrm{x}}{ }^{(0)}, \quad \text { if }\left|\Delta \mathrm{k}_{\mathrm{x}} \mathrm{u}_{\mathrm{x}}(\mathrm{x})\right|<\mu \Delta \mathrm{F}_{\mathrm{z}}=\mu \Delta \mathrm{k}_{\mathrm{z}} \mathrm{u}_{\mathrm{z}}(\mathrm{x}) \\
& \mathrm{u}_{\mathrm{x}}(\mathrm{x})= \pm \frac{\mu \Delta \mathrm{F}_{\mathrm{z}}(\mathrm{x})}{\Delta \mathrm{k}}, \text { in a state of slip. }
\end{aligned}
$$

where $\mathrm{u}_{\mathrm{x}}(\mathrm{x})$ denotes here the horizontal displacement of a spring at point $\mathrm{x}$. The sign of the second equation depends on the direction of the indenter motion. We denote the radius of the stick-area by $\mathrm{c}$ and find:

$$
\mathrm{G}^{*} \mathrm{u}_{\mathrm{x}}^{(0)}=\mu \mathrm{E}^{*}(\mathrm{~d}-\mathrm{g}(\mathrm{c})),
$$

for which the whole contact area is in slip state. Similarly to the normal contact, the force of a spring at point $\mathrm{x}$ inside the contact area equals:

$$
\Delta \mathrm{F}_{\mathrm{x}}(\mathrm{x})=\Delta \mathrm{k}_{\mathrm{x}} \mathrm{u}_{\mathrm{x}}(\mathrm{x})=\mathrm{G}^{*} \mathrm{u}_{\mathrm{x}}(\mathrm{x}) \Delta \mathrm{x} .
$$

If we choose the spring separation distance to be infinitesimal, we get for the tangential force:

$$
F_{x}=G^{*} \int_{-a}^{a} u_{x}(x) d x
$$


The distribution of tangential stress in the original three-dimensional contact can be calculated according to [2]:

$$
\tau_{\mathrm{zr}}(\mathrm{r})=-\frac{\mathrm{G}^{*}}{\pi} \int_{\mathrm{r}}^{\infty} \frac{\mathrm{u}_{\mathrm{x}}^{\prime}(\mathrm{x})}{\sqrt{\mathrm{x}^{2}-\mathrm{r}^{2}}} \mathrm{dx} .
$$

For our problem, we choose the classical contact between a sphere with radius $\mathrm{R}$ and a plane. The modified profile of $\mathrm{z}=\mathrm{f}(\mathrm{r})=\mathrm{r}^{2} / 2 \mathrm{R}$ is $\mathrm{g}(\mathrm{x})=\mathrm{x}^{2} / \mathrm{R}$. Normal and tangential stiffness follow Eqs. (5) and (6). With a given indentation depth $\mathrm{d}$, we calculate contact radius a according to Eq. (10). The length of the elastic foundation is set to $2 \mathrm{a}$ and consists of $\mathrm{N}_{\mathrm{S}}$ springs. Distance $\Delta \mathrm{x}$ between two adjacent springs is $2 \mathrm{a} / \mathrm{N}_{\mathrm{S}}$. The normal displacement is $u_{z}(x)=d-g(x)=d-x^{2} / R$. For the normal force, we get $F_{N}=2 \sum_{k=1}^{N_{s}} u_{z}(k) E * \Delta x$.

With a given indenter displacement $\mathrm{u}_{\mathrm{x}}{ }^{(0)}$, we first assume that every spring sticks. Next, we check if tangential spring force is exceeding the maximum tangential force according to the first part in Eq. (15) and where required, we adjust the displacement.

For the tangential force, we get $F_{x}=2 \sum_{k=1}^{N_{s}} u_{x}(k) G^{*} \Delta x$.

\section{REDUCTION OF RESIDUAL SHEAR STRESS MAGNITUDE}

We push a spherical indenter into an elastic plane and drag it horizontally. If we drag it not too far to one side, our contact area will be divided in a stick-area in the center and a slip-area on the outside. The stick-area will have the shape of a circle and the slip-area will encircle it in the shape of an annulus. By moving it further in the chosen direction, the stick-area gets smaller and smaller before it disappears completely and we reach macroscopic slipping.

If we move the indenter in one direction without reaching macroscopic slipping and subsequently perform a small displacement in the opposite direction, we will observe that: the former stick-area is still in stick-state. More inner parts of the former slip-area are now also in stick-state. The outermost border of the former slip-area is in slip-state again.

Thus, we will observe different displacement areas: in the center, there will be a circle where the displacement equals the macroscopic displacement of the indenter, then there is an annulus in a stick-state, which was at some former time in a slip-state, and finally on the outermost border there will always be an annulus in slip-state. That is, by simply alternating the direction of the indenter several times, we can create a strongly fragmented displacement field.

If now, for example, we drag the indenter until the whole contact is in slip-state, we could create a growing stick-area with a fragmented displacement field, by simply moving in a certain back-and-forth motion with falling amplitudes. As the displacement field determines the shear stress distribution, we can manipulate the stress by moving the indenter in a given way.

In order to minimize the shear stress, the following procedure is discussed. 
On a rigid plane base, we push a spherical indenter until an indentation depth $\mathrm{d}$ and related normal force $\mathrm{F}_{\mathrm{N}}$ are reached. We keep this configuration for the normal contact throughout the following procedure.

The origin of coordinate $\mathrm{u}_{\mathrm{x}}{ }^{(0)}$ is placed on the rigid plane.

Subsequently we displace the indenter horizontally by $\mathrm{u}_{\mathrm{xmax}}{ }^{(0)}$ in $\mathrm{u}_{\mathrm{x}}{ }^{(0)}$ - direction so that the stick area disappears completely according to Eq. (4) and the indenter reaches macroscopic slipping. Now we drag the indenter back and forth with linearly falling amplitude around the origin of $\mathrm{u}_{\mathrm{x}}{ }^{(0)}$-coordinate.

Let $\mathrm{N}_{\mathrm{R}}$ be the total preselected amount of reversal points and $\mathrm{k}$ the current reversal point, then the analytic expression describing the position of the indenter relative to the rigid plane is:

$$
u_{\mathrm{x}}^{(0)}(\mathrm{k})=\frac{\mathrm{u}_{\mathrm{xmax}}^{(0)}}{\mathrm{N}_{\mathrm{R}}}\left(\mathrm{N}_{\mathrm{R}}+1-\mathrm{k}\right)(-1)^{(\mathrm{k}+1)} \text { for } \mathrm{k} \in\left[1,2, \ldots, \mathrm{N}_{\mathrm{R}}+1\right] .
$$

For example, if we choose $\mathrm{N}_{\mathrm{R}}=1$, the indenter moves to $\mathrm{u}_{\mathrm{xmax}}{ }^{(0)}$ and then back to 0 . If we choose $\mathrm{N}_{\mathrm{R}}=2$, the indenter goes to $\mathrm{u}_{\mathrm{x}}{ }^{(0)}=\mathrm{u}_{\mathrm{xmax}}{ }^{(0)}$, then to $-\mathrm{u}_{\mathrm{xmax}}{ }^{(0)} / 2$ and back to 0 .

Hence, the whole procedure is path-controlled by sequence $u_{x}{ }^{(0)}(k)$. Shear stress $\tau(r)$ and tangential force $F_{x}$ are only evaluated at each reversal point. Note that Eq. (20) only describes these points of the movement. The inertial forces of the indenter are not considered and the problem is seen as quasi-static. Therefore, it does not matter in what exact fashion the indenter moves back and forth. Instead of moving linearly, it might as well follow a sinusoidal movement, as depicted in Fig. 1.

Finally, it should be pointed out that the movement always ends (for $k=N_{R}+1$ ) at starting point $\mathrm{u}_{\mathrm{x}}{ }^{(0)}=0$.

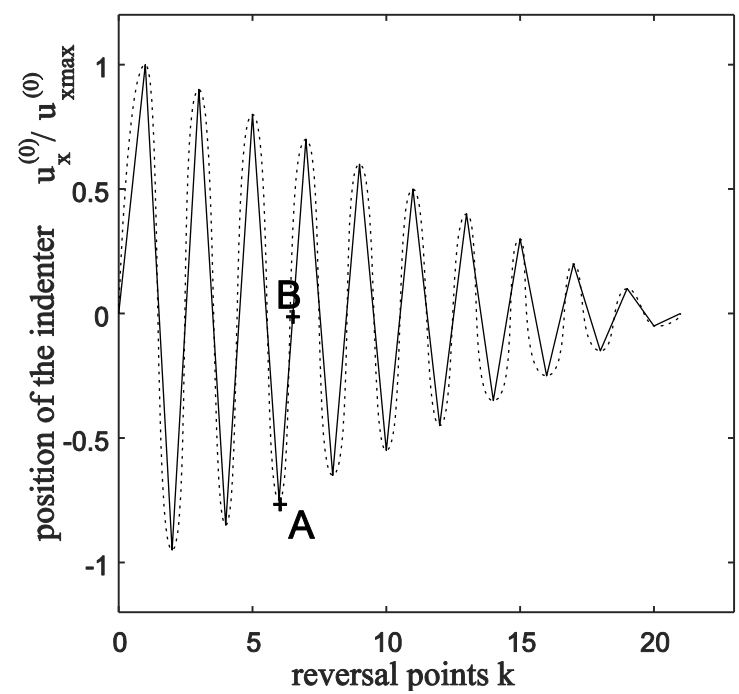

Fig. 1 Normalized position of the indenter on the rigid plane versus the reversal points. $\mathrm{u}_{\mathrm{x} \max }{ }^{(0)}$ is the minimum displacement to reach macroscopic slipping. Total amount of reversal points is 20 . 


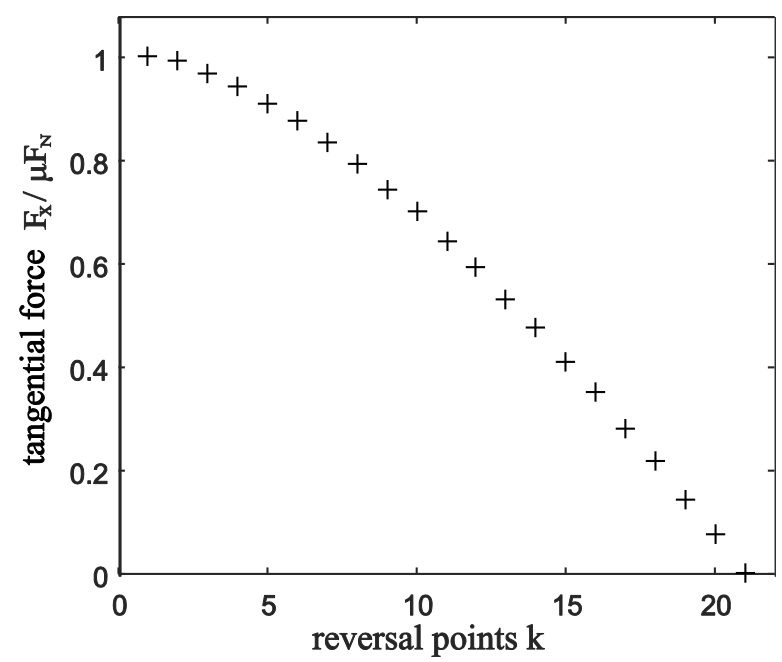

Fig. 2 Normalized tangential force versus reversal points. Total amount $\mathrm{N}_{\mathrm{R}}$ of reversal points is $20 . \mathrm{F}_{\mathrm{N}}$ is the constant normal force

As aforementioned, we have chosen to move the indenter path-controlled. From this, we obtained the tangential forces at each reversal point. Fig. 2 shows the absolute value of the alternating forces. We would get exactly the same motion if we picked our reversal points according to this force sequence. For example the first reversal point $(k=1)$ was defined so that we reach macroscopic slipping, that is: $F_{x}=\mu F_{N}$.

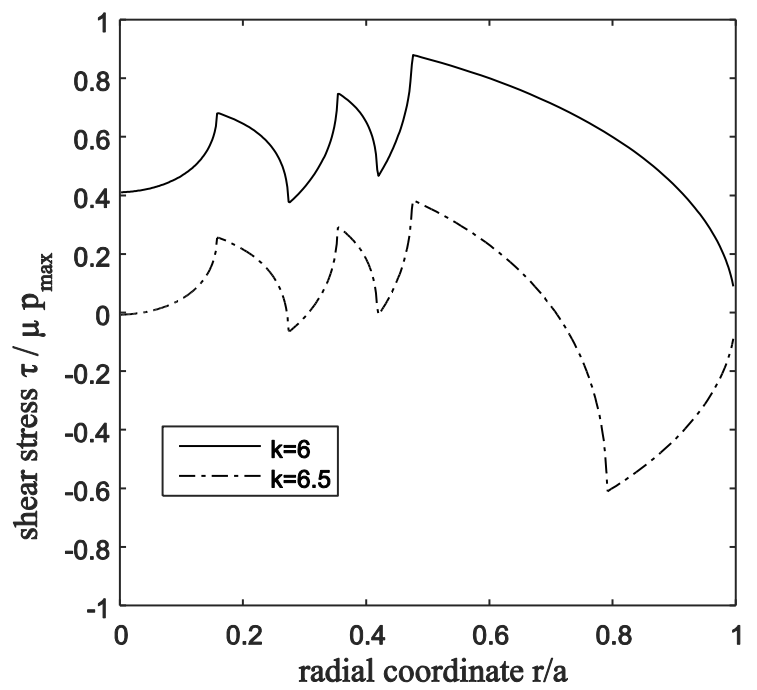

Fig. 3 Shear stress in the contact area after 6 and 6.5 reversal points (A and B in [6]). a is the contact radius and $\mathrm{p}_{\max }$ the maximum normal stress in the contact surface of a spherical indenter and a rigid plane. Total amount $\mathrm{N}_{\mathrm{R}}$ of reversal points is 20 . 
At every state of the movement, we can calculate the shear stress distribution according to Eq. (19). Fig. 3 shows the stress distribution after six back and forth movements (continuous line; point A in Fig. 1), thus exactly at a reversal point. We can see that inside the outer ring of the contact area (approximately $0.5 \mathrm{a}<\mathrm{r}<\mathrm{a}$ ), the shear stress follows $\tau(r)=\mu p(r)$ from which we can conclude that the contact surface in this area is slipping over the plane. In the inside, the shear stress decreases and we have a stick area.

With further back and forth movements at falling amplitude, the stick area will grow more and more towards the edge. The second curve shows the state if we move from the sixth reversal point back to our starting point (dashed line; point B in Fig. 1). Note that there is stick for approximately $r<0.75 \mathrm{a}$. In the outer ring, all points are sliding but the orientation has changed (negative shear stress in the figure).

More generally, we can see that in the MDR space, every spring has a specific maximum tangential deflection. Due to the higher normal deformation, this maximum deflection is greater for the springs in the middle of the contact. With falling amplitude of the movement, little by little those springs enter the stick state, so that they can follow the macroscopic movement of indenter $\mathrm{u}_{\mathrm{x}}{ }^{(0)}$. At the outer edge, the springs can only slightly deform tangentially and are practically always in a slip-state, except shortly after switching orientation at each reversal point.

Fig. 4 shows the shear stress distribution after 20 back-and-forth displacements and return to the origin. As we can see, the residual stress alternates between positive and negative values. That is, the springs are pointing in both directions. It is interesting to see, that the different peaks take almost the same value.

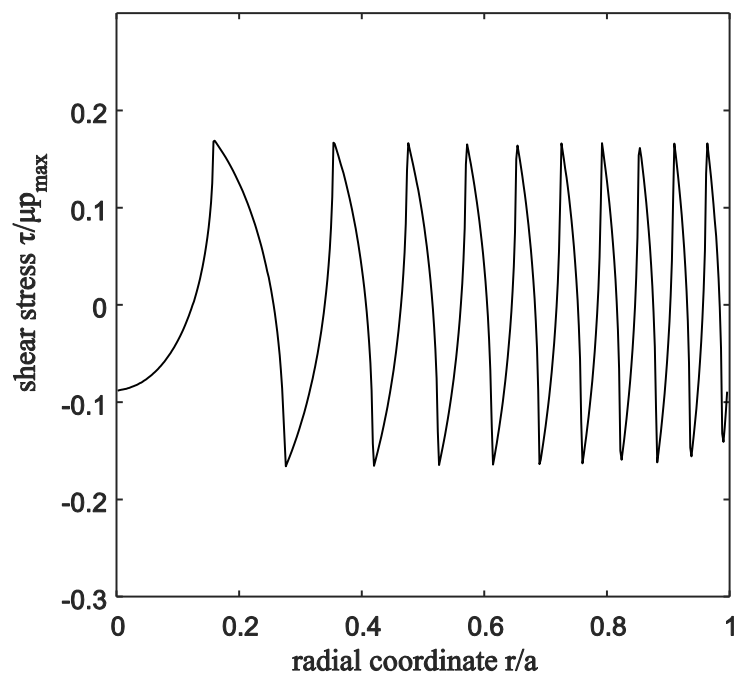

Fig. 4 Shear stress in the contact area after 20 reversal points.

Total amount $\mathrm{N}_{\mathrm{R}}$ of reversal points is 20 
The ultimate purpose of the procedure is to minimize shear stress in the contact area. For this, we can evaluate the shear stress distribution that remains after the dragging as showed Fig. 4. For the evaluation, we want to focus on the extreme values of the stress and calculate therefore the minimum, maximum and arithmetic mean of the absolute values of the shear stress at the end of the dragging. If we do this for different $N_{R}$, we get Fig. 5. As Fig. 4 already illustrated, the peaks are quite evenly and that is why the minima and maxima of the absolute values are close together.

As Fig. 5 further shows, we can easily reduce the shear stress in the contact by moving a few times back and forth. If for example we created the maximum achievable shear stress by reaching macroscopic sliding, then it would be sufficient to invert the initial movement direction twice with falling amplitude according to Eq. (20), to almost halve this stress $\left(\mathrm{N}_{\mathrm{R}}=2\right)$.

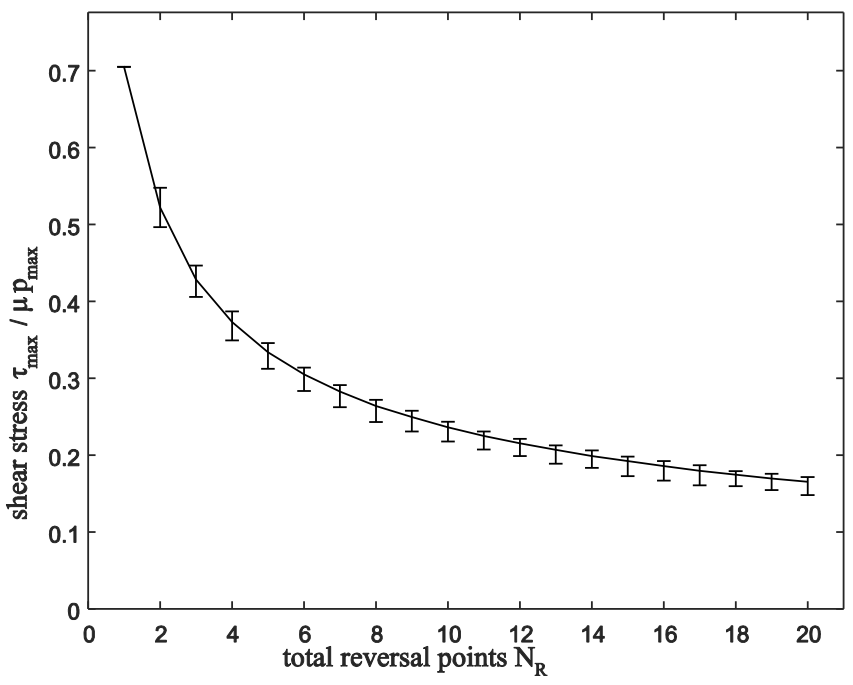

Fig. 5 Minimum, maximum and arithmetic mean of the shear stress at the end of the dragging $\left(k=\mathrm{N}_{\mathrm{R}}+1\right)$ versus different reversal point amounts.

\section{CONCLUSION}

We have analyzed the Hertz contact with the Coulomb friction for the case that the spherical indenter is being moved with falling amplitudes tangentially to a plane. With the aid of the method of dimensionality reduction (MDR), we divided the contact area at every step into a stick and a slip area and subsequently determined the shear stress distribution.

We find that after the movements, the remaining stress has comparable portion in both directions. With respect to the radial coordinate, it changes orientation in close succession. The peaks of this curve are equally large in good approximation. 
The back-and-forth tangential movement proves to be a good way to reduce the residual shear stress inside a frictional contract. With only a few iterations, the maximum stress value can be reduced effectively.

\section{REFERENCES}

1. Hertz, H., 1881, Über die Berührung fester elastischer Körper, Journal für die reine und angewandte Mathematik, 92, pp. 156-171.

2. Popov, V.L., Heß, M., 2015, Method of Dimensionality Reduction in Contact Mechanics and Friction, Springer.

3. Mindlin, R., Mason, W., Osmer, J., Deresiewicz, H., 1952, Effects of an oscillating tangential force on the contact surfaces of elastic spheres, Proc. 1st. US Nat'l. Congr. Appl. Mech. ASME New York, pp. 203-208.

4. Borri-Brunetto, M., Carpinteri, A., Invernizzi, S. \& Paggi, M., 2005, Micro-slip of rough surfaces under cyclic tangential loading, Proceedings of the 4th Contact Mechanics International Symposium, pp. 333-340.

5. Ciavarella, M., 2013, Frictional energy dissipation in Hertzian contact under biaxial tangential harmonically varying loads, J Strain Analysis, 49(1), pp. 27-32.

6. E. Madelung, 1905, Über Magnetisierung durch schnell verlaufende Ströme und die Wirkungsweise des Rutherford-Marconischen Magnetdetektors, Ann. Phys. , 322(10), pp. 861-890.

7. Pohrt, R., Li, Q., 2014, Complete boundary element formulation for normal and tangential contact problems, Physical Mesomechanics, 17/4, pp. 334-340.

8. Popov, V. L., 2014, Analytic solution for the limiting shape of profiles due to fretting wear, Scientific reports, 4, doi:10.1038/srep03749

9. Aleshin, V., Abeele, K. V. D., 2012, Hertz-Mindlin problem for arbitrary oblique 2D loading: General solution by memory diagrams, Journal of the Mechanics and Physics of Solids, 60/1, pp. 14-36.

10. Aleshin, V., Abeele, K. V. D., 2009, Preisach analysis of the Hertz-Mindlin system, Journal of the Mechanics and Physics of Solids, 57, pp. 657-672.

11. Popov, V., Gray, J., 2012, Prandtl-Tomlinson model: History and applications in friction, plasticity, and nanotechnologies, Z. Angew. Math. Mech., 92/9, pp. 683-708.

12. Prandtl, L., 1928, Ein Gedankenmodell zur kinetischen Theorie der festen Körper, Z. Angew. Math. Mech., 8, pp. 85-106. 